\title{
BMJ Open Estimating vaccine confidence levels among healthcare students and staff of a tertiary institution in South Africa: protocol of a cross-sectional survey
}

\author{
Elizabeth O Oduwole (D) , Hassan Mahomed (D) , ${ }^{1}$ Birhanu T Ayele, ${ }^{2}$ \\ Charles Shey Wiysonge (iD) ${ }^{1,3}$
}

To cite: Oduwole EO, Mahomed H, Ayele BT, et al. Estimating vaccine confidence levels among healthcare students and staff of a tertiary institution in South Africa: protocol of a crosssectional survey. BMJ Open 2021;11:e049877. doi:10.1136/ bmjopen-2021-049877

- Prepublication history for this paper is available online. To view these files, please visit the journal online (http://dx.doi. org/10.1136/bmjopen-2021049877).

BTA deceased on 01 February 2021

Received 06 February 2021

Revised 22 April 2021

Accepted 28 April 2021

Check for updates

(C) Author(s) (or their employer(s)) 2021. Re-use permitted under CC BY-NC. No commercial re-use. See rights and permissions. Published by BMJ.

For numbered affiliations see end of article.

Correspondence to

Elizabeth 0 Oduwole;

oduwoleelizabeth@gmail.com

\section{ABSTRACT}

Introduction The outbreak of novel COVID-19 caught the world off guard in the first quarter of 2020. To stem the tide of this pandemic, there was acceleration of the development, testing and prelicensure approval for emergency use of some COVID-19 vaccine candidates. This led to raised public concern about their safety and efficacy, compounding the challenges of vaccine hesitancy. The onus of managing and administering these vaccines to a sceptical populace when they do become available rests mostly on the shoulders of healthcare workers (HCWs). Therefore, the vaccine confidence levels of HCWs become critical to the success of vaccination endeavours. This proposed study aims to estimate the level of vaccine confidence and the intention to receive a COVID-19 vaccine among future HCWs and their trainers at a specific university in Cape Town, South Africa, and to identify any vaccination concerns early for targeted intervention. Methods and analysis This proposed study is a crosssectional survey study. An online questionnaire will be distributed to all current staff and students of the Faculty of Medicine Health Sciences of Stellenbosch University in Cape Town, South Africa. No sampling strategy will be employed. The survey questionnaire will consist of demographic questions (consisting of six items) and vaccine confidence questions (comprising six items in Likert scale format). Log binomial models will be employed to identify factors associated with vaccine confidence and intention. The strength of association will be assessed using the $\mathrm{OR}$ and its $95 \% \mathrm{Cl}$. Statistical significance will be defined at a $p$ value $<0.05$.

Ethics and dissemination Ethics approval has been obtained for the study from Stellenbosch University (Human Research Ethics Committee reference number S19/01/014 (PhD)). The results will be shared with relevant health authorities, presented at conferences and published in a peer-reviewed journal.

\section{INTRODUCTION}

\section{Background}

The world is currently in the grip of COVID-19 pandemic caused by SARS-CoV-2. ${ }^{1}$ The disease has claimed millions of lives and wreaked economic havoc worldwide since it was declared a pandemic by the WHO in March
Strengths and limitations of this study

- The questionnaire will be pilot tested to ensure that questions and response options are understood as intended, and are well adapted to the local context.

- Weighting method will be used to control for nonresponse bias.

- Log binomial regression will be used to identify factors associated with vaccine confidence.

- A limitation of the study is that vaccine confidence will be measured using an adapted questionnaire that has not been formally validated in previous studies.

2020. The problem is further compounded by some countries experiencing a second and third wave of the pandemic ${ }^{23}$ and the emergence of new and possibly more virulent strains of the virus. ${ }^{4}$ All these, coupled with its high infectivity rate, resulted in an unprecedented global effort to develop and produce safe and effective vaccines within an equally unprecedented short time frame. ${ }^{5}$ This feat was achieved in the last quarter of the year 2020 when the first set of safe and effective vaccine candidates received prelicensure emergency use authorisation by the Food and Drug Administration (FDA) in the USA and other countries. ${ }^{6}$

Since then, there has been a gradual roll-out of COVID-19 vaccination with several vaccines by multiple countries. Millions of people have been vaccinated, and many more are scheduled to receive COVID-19 vaccination, raising hopes that the pandemic will soon be brought under control and life can go back to normal.

However, the rapid positive progress in the fight against the COVID-19 pandemic has also brought new concerns. One such concern is the effect of the unprecedented speed of development and emergency use 
authorisation of the successful vaccine candidates on the already tenuous public confidence in vaccines and vaccinations. Despite concerted efforts of some government agencies such as the FDA, ${ }^{7}$ regional health agencies such as the European Medicines Agency, ${ }^{8}$ and the $\mathrm{WHO}^{9}$ and other credible sources ${ }^{510-12}$ to allay these fears and reassure the public that these speedy developments were possible due to years of acquired and accumulated knowledge, and that scientific rigour was not compromised, the public is still cautious of these successful vaccine candidates. $^{10}$

Prior to the pandemic, the waning public confidence in vaccines that had been simmering for decades reached crisis levels, prompting the WHO to declare 'vaccine hesitancy' as one of the 10 threats to global health in the year $2019 .^{13}$ Defined in 2012 as the delay in acceptance or refusal of vaccination despite the availability of vaccination services, ${ }^{14}$ vaccine hesitancy is complex and context specific, and varies across time, place and vaccines. ${ }^{14} 15$ Vaccine confidence is one of the most common determinants of vaccine hesitancy globally. ${ }^{16}$ Vaccine confidence refers to trust in the safety and effectiveness of vaccines, trust in the competency of the healthcare providers who administer them and trust in the intentions of the policymakers who propose them. ${ }^{17}$ Therefore, estimating the vaccine confidence levels in a population should give an indication of how hesitant or otherwise the population may be. This is of particular importance if the vaccine confidence investigation is conducted among the subset of the population charged with the responsibility of administering and promoting vaccines and vaccination to the rest of the general public; that is, healthcare workers and their trainers.

The crucial role of healthcare workers to the success of vaccination uptake is well recognised as they are usually the most trusted source of health information for the general public. ${ }^{18-20}$ Negative vaccine sentiments expressed by some of them are a cause for concern as those selfreporting to be vaccine hesitant also admit to not administering, recommending or following the recommended vaccination schedule with their patients. ${ }^{1820}$ Vaccines such as human papillomavirus (HPV), hepatitis B, seasonal influenza and pandemic influenza vaccines are some of the vaccines that healthcare workers (HCWs) have been known to exhibit hesitancy towards for various reasons such as perceived lack of effectiveness (eg, seasonal influenza vaccines) and doubt about the safety (eg, HPV vaccines) ${ }^{19-22}$ The review of Paterson and her team in 2018 reveals that hesitancy by HCWs towards influenza vaccines was the highest reported vaccine hesitancy in the reviewed literature at $86 \% .{ }^{18}$ Other vaccines that HCWs were hesitant about are hepatitis B, pertussis, smallpox, varicella and $\mathrm{HPV}$ vaccines. A small percentage $(3 \%)$ of the literature reviewed in the same study documented HCW hesitancy towards vaccines in general. ${ }^{18}$ This kind of attitude among HCWs is quite concerning. These attitudes can lead to actions that can undermine vaccination uptake in populations served by such HCWs, leading to pockets of undervaccinated individuals who could potentially serve as reservoirs for outbreaks of vaccine preventable diseases. This is an undesirable situation to be avoided as much as possible, especially in this pandemic period.

\section{Study rationale}

Vaccine attitudes of HCWs have been investigated in diverse contexts and places, ${ }^{192-26}$ and similar studies have been carried out among medical students and HCWs in training. ${ }^{27-32}$ However, few studies have reported on vaccine attitudes among HCWs in training and their trainers together. This underscores the need for such a study as this one that proposes to estimate the vaccine confidence index among both subsets of the HCW population. In addition, the study will also be providing insight into intentions to take COVID-19 vaccines in the study population when they become available. This will be a crucial piece in the knowledge puzzle of the vaccination attitudes in this population, and also contribute to the knowledge of vaccination attitudes of the wider South African population. In the planned three-phase roll-out of COVID-19 vaccination in the country, ${ }^{33}$ front-line HCWs are the priority population scheduled to receive the vaccines in the first phase. Having this baseline knowledge will assist in identifying possible barriers and facilitators to the receipt of the COVID-19 vaccines in the study population, and will provide more current data on intention to take COVID-19 vaccines of the general South African population in addition to those previously reported. ${ }^{1634}$

In a 67-country survey study of the state of vaccine confidence conducted by Larson and her team they found that vaccine sentiments were generally positive across the countries surveyed which included South Africa. ${ }^{16}$ However, they also reported a diversity of negative sentiments regarding vaccine safety and other issues which varied between continents and countries. The study had four statements related to attitudes towards vaccines: 'vaccines are important for children to have'; 'overall I think vaccines are safe'; 'overall I think vaccines are effective'; and 'vaccines are compatible with my religious beliefs'. Participants were asked to rate the degree to which they agree with each statement on the 5-point Likert scale: 'strongly agree', 'tend to agree', 'do not know', 'tend to disagree' or 'strongly disagree'. The proportion of South African participants who were sceptical of the importance, safety, effectiveness and religious compatibility of vaccines was $6.6 \%, 10.2 \%, 10.2 \%$ and $14.2 \%$, respectively. Over half a decade has elapsed since this survey was conducted, and the world at large has changed and so has the vaccination landscape, especially with the advent of SARS-CoV-2 and the COVID-19 pandemic.

Therefore, it is important and imperative to explore what changes might have occurred over this period of time and what effect it might have on vaccine confidence of South Africans as the country prepares for its 
COVID-19 vaccination roll-out of which the HCWs are the scheduled priority recipients in phase I. ${ }^{33}$

HCWs in training are future healthcare practitioners, trainers and policymakers. As such their vaccine attitudes need to be measured and optimum attention given to them while they are still in training to ensure that a positive vaccination attitude is developed and maintained during their training that will hopefully last long into their professional lives. Therefore, measuring their vaccine confidence is critical to developing targeted interventions that will address potential vaccine concerns that they may be harbouring. The attitudes of those who provide training to these future healthcare practitioners are also key to any intervention which seek to foster positive attitudes to vaccines among this group. After due consideration of all the cogent reasons proffered above, and the potential benefits inherent in this proposed study, the need for such a study at such a time as this cannot be overemphasised.

\section{Study aim}

The proposed study seeks to answer the following research question: what is the level of vaccine confidence of a cohort of future healthcare workers and their trainers at an academic institution in Cape Town, South Africa? The aim is to estimate the vaccine confidence level of current academic staff and students of the Faculty of Medicine and Health Sciences at Stellenbosch University in Cape Town. Additionally, we will estimate the effect of several predictors on the vaccine confidence level in this population.

\section{METHODS AND ANALYSIS \\ Study design}

This is a cross-sectional survey targeting all academic staff and students of the selected institution. The operational definition of 'academic staff' for the purpose of this proposed study is staff that are engaged in research or teaching at undergraduate and/or postgraduate levels. Voluntary participation in the survey will be deemed as consent. Only current academic staff and students are eligible to participate.

\section{Study population and sample size}

The population was defined as the current (as at the time of commencement of the study) staff and students, and individuals who are both staff and students of the Faculty of Medicine and Health Sciences of Stellenbosch University in Cape Town, South Africa. All members of the core academic population of the institution will be invited to take part in the survey. Therefore, no sampling strategy will be applied, and the actual sample size will be determined by the response rate, nevertheless, we will propose a tentative sample size.

Methods to increase the response rate such as using a succinct questionnaire, weekly email reminders and incentive (lucky draws) will be used to encourage optimum participation in the survey. It is anticipated that this will result in an unbiased, representative sample.

\section{Sample size}

The sample size is estimated based on $64 \%$ vaccine confidence (actual figures from a recent survey ${ }^{35}$ ) of a finite population of between 3500 and 5000 potential participants (an educated guess of the total number of potential participants). An estimated sample size of 1009 is calculated to produce a two-sided $95 \%$ CI with a precision (half width) of 0.025 when the actual proportion is near 0.6400 and the estimated population size is 3500 . Similarly, $\mathrm{n}=1105$ when the estimated population size is 5000 . Therefore, depending on the actual population size, a sample size of between 1009 and 1105 is required for this study.

\section{Data collection}

The survey will be conducted online using Checkbox survey software on the Stellenbosch University Survey platform to capture participants' responses, and no sampling will be done. The target population is the entire current academic staff and students of the Faculty of Medicine and Health Sciences of Stellenbosch University (ie, a census of all potential participants). It is expected that enough data would be generated through the responses received to power the study to answer the research question. A succinct questionnaire designed purposively for the planned study will be the tool of data collection. It will consist of two types of questions: (1) demographic information which includes age, gender, religion and number of years of post-high school schooling to serve as covariates in the regression analysis of the vaccine sentiments; and (2) the four vaccine confidence statements used in the 67 -country survey, ${ }^{16}$ with the addition of two new statements. The four vaccine confidence statements have been used in several previous studies ${ }^{16}$ but it is unclear if they have been validated. ${ }^{36}$ The four vaccine confidence statements from the 67-country study are: 'vaccines are important for children to have'; 'overall I think vaccines are safe'; 'overall I think vaccines are effective'; and 'vaccines are compatible with my religious beliefs'. We added two new statements to the questionnaire. The first of the two additional statements is about the importance of own vaccination, that is, 'vaccines are important for me to have'. The second statement is about COVID-19 vaccination intention, that is, 'I will take a COVID-19 vaccine when one becomes available'.

The two additional questions were developed in consultation with the study supervisors, who are experts in the field of vaccines. The rationale for the first additional statement is to investigate the participant's personal vaccine attitude and to check the variation between an individual's vaccine sentiment and that expressed for their dependents. It has been documented in the literature that sometimes there are differences in an individual's willingness to vaccinate and their willingness to vaccinate their dependents. ${ }^{37}$ The rationale for the 
second question is to gauge the willingness of the study participants to receive COVID-19 vaccines especially as the country is at an advanced stage of preparation for the COVID-19 vaccine roll-out. The inclusion of these two new statements in this vaccine confidence estimation study is informed by the expert opinion of the study supervisors as earlier mentioned, and no specific theoretical framework will be applied in this study.

The full questionnaire will be pilot tested before the commencement of the survey in order to establish feasibility, face validity and logistics. All participants will be encouraged to answer all questions honestly. For each vaccine confidence statement, each participant will be asked to rate the degree with which he or she agrees with it; on a 5-point Likert scale: strongly agree, tend to agree, do not know, tend to disagree, strongly disagree. The questionnaire will be in English.

\section{Data analysis plan}

After data collection, each questionnaire will be checked for its completeness. Data entry, cleaning and coding will be done using Microsoft Excel program and exported to Stata software V.16.1 (College Station, Texas) or any other appropriate software for analysis. Categorical variables will be summarised using frequencies and proportions. Continuous covariates will be presented using means and SDs if normally distributed or using medians and IQR if not normally distributed.

The $\chi^{2}$ test (for categorical variables) and Student's t-test or analysis of variance (for continuous variables) will be used to assess the association between vaccine confidence or intention and potential predictors. The log binomial regression model will be used if the outcome is common ( $>10 \%$ vaccine acceptance). This is expected to be the case based on the previous survey earlier mentioned. ${ }^{35}$ This will be employed to identify factors associated with vaccine confidence and intention. The outcome of vaccine acceptance will be regressed against independent variables such as age, gender, number of years of postmatric education, religion, highest degree attained and academic status (ie, whether staff, student or both).The strength of association will be assessed using the OR and its $95 \%$ CI. Statistical significance will be defined at a $\mathrm{p}$ value $<0.05$. The main outcome to be investigated is the variability in the vaccine sentiment and vaccine intention within and across all groups. This will be done by considering the fraction of respondents that will either agree or disagree with the five statements on immunisation and the one statement on 'intention to vaccinate' previously described. ${ }^{16}$ The 'strongly agree' and 'tend to agree' responses will be combined to make up the positive vaccine sentiment variable, while the 'strongly disagree' and 'tend to disagree' responses will be combined to make up the negative vaccine sentiment variable. The 'don't know response' and no response will be removed from the data prior to analyses.

\section{Patient and public involvement}

Patients and the public are not involved in the design, execution and analysis of the study.

\section{LIMITATIONS}

A limitation of this proposed study is a possible low response rate which is an inherent limitation of such online surveys. To mitigate against this, the questionnaire is kept short (a total of 12 items in all), drop-down options or multiple-choice answers are provided as appropriate to facilitate obtaining timeous, complete and correct responses. At least two reminders, one per week, will be sent to follow-up 2 weeks after the initial email with the survey link is circulated.

Another possible limitation may be responders' bias due to the voluntary nature of the instrument. Some responders may not want to, or honestly disclose their true vaccine sentiments. To counter this limitation, responders will be assured in the survey invite email of guaranteed confidentiality and anonymity of their responses, and the null negative consequences of their participation or otherwise in the survey.

It is also possible that respondents who are less confident with respect to vaccines may be less likely to respond to this questionnaire. A weighting method would be used to adjust for non-response bias, should this challenge be encountered. ${ }^{38}$

\section{ORIGINALITY AND ANTICIPATED IMPACT OF THE PROPOSED STUDY}

The originality of this study lies in the fact that to the best of our knowledge, no such study has been conducted among the staff and students of the Faculty of Medicine and Health Sciences at this specific University. Anticipated study impact includes but is not limited to: the generation of a baseline knowledge of vaccine confidence level of the study population, identifying vaccine confidence issues that are of concern among future healthcare professionals and their trainers, and all the other benefits previously mentioned. This would assist in laying the foundation for the development of tailored interventions to address such concerns by responsible authorities. Moreover, the knowledge generated by this proposed study would add to the growing body of available literature on the potential issues that future healthcare workers may face and provide evidence to mitigate them while they are still in training.

\section{ETHICS AND DISSEMINATION}

This study obtained ethics approval from Stellenbosch University in South Africa: Human Research Ethics Committee reference number S19/01/014 (PhD). Participation in the survey will be voluntary. The right to participate or decline, and the offer of cash incentives of 500 South African rands (to be given to three randomly selected participants) will be clearly detailed in the survey invite email. Participation in the survey is deemed as consent.

The study results will be presented at conferences and other relevant and appropriate platforms. It will also be published in a peer-reviewed journal. 


\section{Author affiliations}

${ }^{1}$ Division of Health Systems and Public Health, Department of Global Health, Faculty of Medicine and Health Sciences, Stellenbosch University, Stellenbosch, Western

Cape, South Africa

${ }^{2}$ Division of Epidemiology and Biostatistics, Department of Global Health, Faculty of Medicine and Health Sciences, Stellenbosch University, Stellenbosch, Western Cape, South Africa

${ }^{3}$ Cochrane South Africa, South African Medical Research Council, Tygerberg, South Africa

\section{Twitter Charles Shey Wiysonge @CharlesShey}

Acknowledgements The statistical input, constructive feedback and promptness of response of BTA are posthumously acknowledged by the surviving three authors. We also acknowledge the efforts of Ms Tonya Esterhuizen who took over the statistical duties of BTA at such short notice in trying times.

Contributors EOO led the conceptualisation, design, and drafted the protocol. BTA collaborated on the data analysis plan and gave feedback on the manuscript. HM and CSW provided the supervisory overview and feedback on the overall study, methodology and the manuscript. This team of four authors gives their approval for the publishing of this protocol manuscript.

Funding The authors acknowledge the funding received from the South African Medical Research Council (SAMRC) in the form of: (A) a student bursary to E00 through the SAMRC Internship Scholarship programme (SAMRC project code: 57020), and (B) funding for the open access publication costs for this manuscript through Cochrane South Africa, an SAMRC intramural research unit.

Competing interests None declared.

Patient and public involvement Patients and/or the public were not involved in the design, or conduct, or reporting, or dissemination plans of this research.

Patient consent for publication Not required.

Provenance and peer review Not commissioned; externally peer reviewed.

Open access This is an open access article distributed in accordance with the Creative Commons Attribution Non Commercial (CC BY-NC 4.0) license, which permits others to distribute, remix, adapt, build upon this work non-commercially, and license their derivative works on different terms, provided the original work is properly cited, appropriate credit is given, any changes made indicated, and the use is non-commercial. See: http://creativecommons.org/licenses/by-nc/4.0/.

\section{ORCID iDs}

Elizabeth 0 Oduwole http://orcid.org/0000-0002-7192-242X

Hassan Mahomed http://orcid.org/0000-0001-6775-9482

Charles Shey Wiysonge http://orcid.org/0000-0002-1273-4779

\section{REFERENCES}

1 Listings of WHO's response to COVID-19. Available: https://www. who.int/news/item/29-06-2020-covidtimeline [Accessed 16 January 2021].

2 Analytical report of the first, second and third wave studies, 2020. Available: https://www.unicef.org/georgia/media/4736/file/COVID-19Study-Analytical-Report-1-st-2nd-and-3rd-waves-Eng.pdf\#page= $12 \&$ zoom $=100,92,96$

3 NICD. COVID-19 second wave in South Africa, 2020. Available: https://www.nicd.ac.za/covid-19-second-wave-in-south-africa/ [Accessed 16 January 2021].

4 A new strain of coronavirus: what you should know | Johns Hopkins medicine. Available: https://www.hopkinsmedicine.org/health/ conditions-and-diseases/coronavirus/a-new-strain-of-coronaviruswhat-you-should-know [Accessed 16 January 2021].

5 Lurie N, Saville M, Hatchett R, et al. Developing Covid-19 vaccines at pandemic speed. N Engl J Med 2020:382:1969-73.

6 FDA. Emergency Use Authorization | FDA. Food \& Drug Administration, 2020. Available: https://www.fda.gov/emergencypreparedness-and-response/mcm-legal-regulatory-and-policyframework/emergency-use-authorization [Accessed 16 January 2021].

7 FDA. COVID-19 vaccines | FDA. food and drug administration, 2021. Available: https://www.fda.gov/emergency-preparedness-andresponse/coronavirus-disease-2019-covid-19/covid-19-vaccines [Accessed 6 March 2021].
8 EMA. COVID-19 vaccines: development, evaluation, approval and monitoring | European medicines Agency, 2020. Available: https:// www.ema.europa.eu/en/human-regulatory/overview/public-healththreats/coronavirus-disease-covid-19/treatments-vaccines/vaccinescovid-19/covid-19-vaccines-development-evaluation-approvalmonitoring [Accessed 6 March 2021].

9 WHO. Coronavirus disease (COVID-19): vaccines. WHO, 2020. Available: https://www.who.int/news-room/q-a-detail/coronavirusdisease-(covid-19)-vaccines [Accessed 27 March 2021].

10 How were researchers able to develop COVID-19 vaccines so quickly? - UChicago Medicine. Available: https://www. uchicagomedicine.org/forefront/coronavirus-disease-covid-19/ developing-covid19-vaccines-quickly [Accessed 6 March 2021].

11 Krause PR, Gruber MF. Emergency Use Authorization of Covid Vaccines - Safety and Efficacy Follow-up Considerations. N Engl J Med 2020;383:e107.

12 Stratford J, MacKenzie E, Mockford E. Balancing speed and safety: the Authorisation of Covid-19 vaccines and medicines. Judicial Review 2020;25:105-17.

13 WHO. Ten threats to global health in 2019. World Health Organisation, 2019: 1-18.

14 MacDonald NE, Eskola J, Liang X, SAGE Working Group on Vaccine Hesitancy. Vaccine hesitancy: definition, scope and determinants. Vaccine 2015;33:4161-4.

15 Betsch C, Böhm R, Chapman GB. Using behavioral insights to increase vaccination policy effectiveness. Policy Insights from Behav Brain Sci 2015;2:61-73.

16 Larson HJ, de Figueiredo A, Xiahong Z, et al. The state of vaccine confidence 2016: global insights through a 67-Country survey. EBioMedicine 2016;12:295-301.

17 Eskola J, Duclos P, Schuster M, et al. How to deal with vaccine hesitancy? Vaccine 2015;33:4215-7.

18 Paterson P, Meurice F, Stanberry LR, et al. Vaccine hesitancy and healthcare providers. Vaccine 2016;34:6700-6.

19 Karafillakis E, Dinca I, Apfel F, et al. Vaccine hesitancy among healthcare workers in Europe: a qualitative study. Vaccine 2016;34:5013-20.

20 Verger P, Fressard L, Collange F, et al. Vaccine Hesitancy among general practitioners and its determinants during controversies: a national cross-sectional survey in France. EBioMedicine 2015;2:891-7.

21 Yaqub O, Castle-clarke S, Sevdalis N. Social Science \& Medicine Attitudes to vaccination : A critical review. Soc Sci Med 2014;112:1-11.

22 Maltezou HC, Maragos A, Katerelos P, et al. Influenza vaccination acceptance among health-care workers: a nationwide survey. Vaccine 2008;26:1408-10.

23 Suryadevara M, Handel A, Bonville CA, et al. Pediatric provider vaccine hesitancy: an under-recognized obstacle to immunizing children. Vaccine 2015;33:6629-34.

24 MacDonald NE, Dubé E. Unpacking vaccine Hesitancy among healthcare providers. EBioMedicine 2015;2:792-3.

25 Oria PA, Matini W, Nelligan I, et al. Are Kenyan healthcare workers willing to receive the pandemic influenza vaccine? results from a cross-sectional survey of healthcare workers in Kenya about knowledge, attitudes and practices concerning infection with and vaccination against 2009 pandemic influenza A (H1N1), 2010. Vaccine 2011;29:3617-22.

26 Le Maréchal M, Collange F, Fressard L, et al. Design of a national and regional survey among French general practitioners and method of the first wave of survey dedicated to vaccination. Med Mal Infect 2015;45:403-10.

27 Wibabara Y, Banura C, Kalyango J, et al. Hepatitis B vaccination status and associated factors among undergraduate students of Makerere University College of health sciences. PLoS One 2019;14:e0214732-9.

28 Kernéis S, Jacquet C, Bannay A, et al. Vaccine education of medical students: a nationwide cross-sectional survey. Am J Prev Med 2017;53:e97-104.

29 Gallone MS, Gallone MF, Cappelli MG, et al. Medical students' attitude toward influenza vaccination: results of a survey in the University of Bari (Italy). Hum Vaccin Immunother 2017;13:1937-41.

30 Klewer J, Sasnauskaite L, Pavilonis A. Vaccinations in health care students from Germany, Iran, Lithuania and Spain. In: Vaccinations: types, potential complications and health effects, 2020: 25-43.

31 Böhme M, Voigt K, Balogh E, et al. Pertussis vaccination status and vaccine acceptance among medical students: multicenter study in Germany and Hungary. BMC Public Health 2019;19:N.PAG.

32 Loulergue $P$, Launay O. Vaccinations among medical and nursing students: coverage and opportunities. Vaccine 2014;32:4855-9. 
33 All You Need To Know About COVID-19 And Vaccines (pdf Guide) SA Corona Virus Online Portal. 2021; 13.

34 Lazarus J V, Ratzan SC, Palayew A. A global survey of potential acceptance of a COVID-19 vaccine. Nat Med 2020.

35 Boyon $\mathrm{N}$. Three in four adults globally say they would get a vaccine for COVID-19. Ipsos, 2020. Available: https://www.ipsos.com/en-za/ three-four-adults-globally-say-they-would-get-vaccine-covid-19 [Accessed 6 March 2021].
36 Larson HJ, Jarrett C, Schulz WS, et al. Measuring vaccine hesitancy: the development of a survey tool. Vaccine 2015;33:4165-75.

37 Killian M, Detoc M, Berthelot P, et al. Vaccine hesitancy among general practitioners: evaluation and comparison of their immunisation practice for themselves, their patients and their children. Eur J Clin Microbiol Infect Dis 2016;35:1837-43.

38 Prince M. Non-Response bias. in: core psychiatry, 2012. Available: https://www.sciencedirect.com/topics/nursing-and-healthprofessions/nonresponse-bias/pdf 